\title{
Situación del tratamiento trombolítico del infarto agudo de miocardio en los Servicios de Urgencias de Cataluña
}

\author{
F. EPELDE Y GRUPO DE TRABAJO DE CARDIOPATÍA ISQUÉMICA DE LA \\ SOCIEDAD ESPAÑOLA DE MEDICINA DE URGENCIAS (SEMES)
}

\section{RESUMEN}

Objetivo: El objetivo del presente estudio es conocer la situación del tratamiento trombolítico en Cataluña.

Material y método: Estudio retrospectivo enviando un cuestionario a todos los hospitales catalanes para conocer la administración del tratamiento trombolítico.

Resultados: 29 centros contestaron a el cuestionario, lo que supone una población atendida de 4,4 millones de personas y un número total de infartos anuales de 2.853. El tiempo desde el inicio de los síntomas hasta la llegada al hospital fue de $176 \pm 96 \mathrm{~min}$, y un tiempo puerta-aguja de $78,7 \pm 73,8 \mathrm{~min}$. La persona que decide mayoritariamente la administración del trombolítico es el médico de urgencias (17 centros). Un total de $47 \pm 12 \%$ de los IAM recibió tratamiento trombolítico, de los cuales el $61,76 \pm 42,77$ se administraron en el servicio de urgencias. El retraso interno para la administración del tratamiento fue de $33,7 \pm 11,9$ min en los centros sin UCI mientras que en los centros con UCI fue de $112 \pm 8$ $\min (p=0,0067)$. En los centros que disponen de UCI, pero en los cuales el medico de urgencias toma la decisión de la adminstración del trombolítico $(\mathrm{n}=7)$ el retraso interno fue menor $(84,3 \pm 80,59 \mathrm{~min})$.

Conclusiones: El retraso para la administración del tratamiento trombolítico es menor en los centros que no disponen de UCI. Si la decisión de administración del trombolítico es tomada por un médico de urgencias, el retraso en el inicio del tratamiento es menor.

PALABRAS CLAVE: Tratamiento trombolítico. Infarto agudo de miocardio.

\section{SITUATION OF THROMBOLITIC INFARCTION TREATMENT OF PATIENTS WITH ACUTE MYOCARDIAL INFARCTION IN THE EMERGENCY DEPARTMENTS}

\begin{abstract}
Objectives: The aim of the present study was to know the situation of trombolysis in Catalonia.

Material and methods: A questionnaire was sent to all the hopitals in Catalonia regarding to the administration of thrombolitic treatment in their hospitals

Results: 29 centres replied (18 primary, 10 secondary and 1 ter tiary), serving a total catchment population of 4.2 million and with a total number of 2,853 AMIs in. The mean time from the onset of symp toms to arrival at hospital was $176.3 \pm 96 \mathrm{~min}$ and from arrival of the

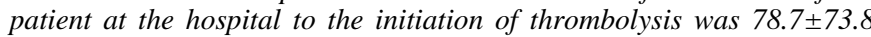
min. Most commonly, it was the Emergency Department staff who took the decision on thrombolysis (17 centres). A total of $47 \pm 12 \%$ of the AMIs received thrombolytic treatment with $61.76 \pm 42.77 \%$ of these treatments being carried out in the Emergency Departments. The internal delay to initiating thrombolysis was of $33.7 \pm 11.9 \mathrm{~min}$ in the centres without an $I T U$ versus $112 \pm 8$ min in those centres with an ITU $(p=0.0067)$. In the centres with an ITU but in which it was the doctor in the Emergency Department who took the decision on thrombolysis $(n=7)$, the internal delay was shorter $(84.3 \pm 80.59 \mathrm{~min})$.

Conclusions: There is a shorter delay in the administration of throm bolytic treatment in those emergency departments which do not have access to an ITU or Coronary Unit than in the Emergency Departments which do have this access. If the decision to carry out thrombolysis is taken by the emergency department doctors there is a substantial reduction in the time to its administration.
\end{abstract}

KEY WORDS: Thrombolitic treatment. Acute myocardial infarction.

Epelde Gonzalo F y Grupo de Trabajo de Cardiopatía Isquémica. Sociedad Española de Medicina de Urgencias (SEMES). Situación del trata miento trombolítico del infarto agudo de miocardio en los Servicios de Urgencias de Cataluña. An Med Interna (Madrid) 2003; $20: 75-77$.

\section{INTRODUCCIÓN}

La administración de fármacos fibrinolíticos ha supuesto un innegable progreso en el tratamiento del infarto agudo de miocardio (IAM). La eficacia del mismo, depende básicamen- te de la precocidad de administración, en este sentido, el estudio GUSTO (1) ha estimado que por cada hora de retraso en iniciar la administración del trombolítico se dejan de salvar 10 vidas por 1.000 pacientes tratados. En el mismo sentido, el estudio MITI (2) refleja que el tiempo de retraso fue un factor

Trabajo presentado en parte en el ACEP RESEARCH FORUM (octubre 23, 2000) Philadelphia.

Trabajo aceptado: 18 de octubre de 2002

Correspondencia: Francisco Epelde Gonzalo. C/ Vinyals, 165, 2º-1. 08221 Terrassa, Barcelona. 
predictivo significativo de mortalidad precoz en el IAM, con una odds ratio de 1,1 por cada hora de retraso.

Son muchos los trabajos que analizan la demora en la administración del tratamiento trombolítico en el IAM (3-5), pero lo que llama la atención, es que pese a la existencia de guias de actuación perfectamente estandarizadas $(6,7)$ exista una gran variabilidad en el manejo de esta patología. En España existen trabajos a este respecto. El estudio PRIAMHO (8) valora el problema de las demoras en la administración del tratamiento trombolítico solo en centros que disponen UCI o Unidad Coronaria, lo que a nuestro entender ofrece una visión parcial del tratamiento IAM en nuestro país, ya que no todos los centros españoles que atienden al IAM disponen de estas áreas tecnificadas.

El objetivo de nuestro trabajo fue conocer la situación del tratamiento al IAM en Cataluña (una región autonómica situada en el NE de España, con 6 millones de habitantes) incluyendo a todos los hospitales implicados y no tan solo a aquellos que disponen de UCI o Unidad Coronaria.

\section{MATERIAL Y MÉTODOS}

Se envió una encuesta a los 40 responsables de Servicios hospitalarios de Urgencias de toda Cataluña que atienden a pacientes afectos de infarto agudo de miocardio. La encuesta fue anónima. Se les solicitó una revisión de sus archivos correspondientes a los pacientes atendidos por un infarto agudo de miocardio durante el último año 1999. Los datos solicitados fueron demográficos (población de referencia atendida, número de IAM anuales), datos referentes a los tiempos de atención al paciente (tiempo desde el inicio de la clínica hasta la llegada al hospital, retraso de administración del trombolítico una vez el enfermo ha llegado al hospital) porcentaje de IAM que reciben trombolisis y tipo de trombolítico utilizado, datos de infraestructura (tipo de hospital, existencia de unidad coronaria o UCI general y cardiólogo de guardia) persona que decide en última instancia el tratamiento trombolítico y lugar donde se administra éste.

\section{RESULTADOS}

Respondieron 29 centros (18 de primer nivel, 10 de segundo nivel y 1 de tercer nivel). La media de urgencias anuales atendidas por estos centros fue de 63,750 $\pm 74,000$ urgencias anuales (6 centros de 25.000 a 52.000 urgencias/año, 9 centros con 52.001-75.000 urgencias/año, 9 centros con 75.000120.000 urgencias/año y 7 ,centros con 120.001 a 300.000 urgencias anuales). En conjunto, estos hospitales cubren una población total de 4,2 millones de habitantes (aproximadamente el $68 \%$ de la población total de Catalunya) y un total de urgencias anuales atendidas de 1.655.000. El número total de IAM en el período estudiado fue de 2.853 (tasa de IAM por $\mathrm{mil} /$ habitantes y año de 1,42; 109,7 \pm 88 ,9 IAM anuales atendidos por centro, rango 9-310 IAM/año). 10 de los hospitales no disponen de UCI, 15 tienen UCI polivalente y 4 Unidad Coronaria independiente.

El tiempo medio desde el inicio de la clínica hasta la llegada al hospital fue de 176,3 $\pm 96 \min$ (rango 60-483 min) y el tiempo desde la llega del enfermo al hospital hasta el inicio de la trombolisis (tiempo puerta aguja) fue de 78,7 $\pm 73,8$ minutos (rango 30-300 $\mathrm{min}$ ). La persona que decide la trombolisis es mayoritariamente el personal de urgencias (17 centros), seguida del intensivista ( 5 centros) y cardiólogo ( 7 centros). Un total de $47 \pm 12 \%$ de los IAM atendidos recibieron tratamiento trombolítico, de éstos, el porcentaje de trombolisis practicadas en urgencias fue del $61,76 \pm 42,77 \%$ (menor al $25 \%$ en 12 centros, del 25 al $50 \%$ en 2 centros, del 50 al $75 \%$ en 2 centros y del 75 al $100 \%$ en 2 centros). El trombolitico mayormente utilizado fue la SK $(67,17 \pm 27,2 \%$ de los casos). No se encontró diferencia estadísticamente significativa en el tiempo de llegada a urgencias entre los centros que no tienen UCI o Coronaria y los que si lo tienen $(174 \pm 85 \mathrm{~min}$ vs $168 \pm 98 \mathrm{~min}$ respectivamente). El tiempo puerta-aguja fue de $33,7 \pm 11,9$ min. en los centros sin UCI frente a $112 \pm 8$ min en los que si tienen UCI $(p=0,0067)$. No se observaron diferencias estadísticamente significativas entre el porcentaje de trombolisis $(46,7 \pm 22,9$ vs $42,8 \pm 20)$ ni el trombolítico mayoritariamente utilizado (Streptokinasa $78,8 \pm 17 \%$ vs $61,7 \pm 29,3 \%$ y r-TPA en $18,7 \pm 36,6 \%$ entre los centros sin UCI y los que si la tienen respectivamente). El $61,7 \pm 43 \%$ de las trombolisis se practicó en el servicio de urgencias. En los centros con UCI en los que el médico de urgencias decide la trombolisis $(n=7)$ ésta se administra más rápidamente $(84,3 \pm 80,59 \mathrm{~min})$ que en los que decide el intensivista $(\mathrm{n}=5)(146 \pm 65 \mathrm{~min})$ o el cardiologo $(\mathrm{n}=7)(115 \pm 89 \mathrm{~min})$, aunque la diferencia no es estadísticamente significativa.

\section{DISCUSIÓN}

La importancia de reducir el tiempo desde el inicio de los síntomas del IAM hasta el comienzo del tratamiento trombolítico es fundamental. Los retrasos en la administración del tratamiento pueden ocurrir desde que el paciente tiene las primeras manifestaciones clínicas de dolor precordial hasta que acude al hospital (retraso prehospitalario), y desde que el enfermo ingresa en el servicio de urgencias hasta que el enfermo recibe el tratamiento trombolítico (tiempo puerta-aguja).

En nuestro estudio, el tiempo de retraso prehospitalario fue similar y sin signigicación estadística entre los centros con UCI o Unidad Coronaria y los que no la tienten. Es un retraso bastante superior al publicado en series norteamericanas (9) e inglesas (10). Estos tiempos de retraso serían susceptibles de mejora mediante campañas de concienciación a la población general, para favorecer que acudan lo antes posible a los servicios de urgencias hospitalarios para recibir atención. Así mismo, la potenciación de la trombolisis prehospitalaria jugará un papel importante en la disminución del tiempo de administración del trombolítico tal y como ha sido demostrado en otros estudios (11).

No existió una diferencia estadísticamente significativa en el porcentaje de trombolisis entre los centros que disponen de UCI o Unidad Coronaria y los que no disponen de ellas, aunque se evidencia una tendencia mayor de trombolisis en los centros sin UCI, que quizá podría ser debida a que al tener un menor retraso interno, algunos enfermos podrían beneficiarse de la trombolisis al estar dentro del período de efectividad del tratamiento. El resultado más interesante del estudio a nuestro entender, es la gran diferencia de tiempo de retraso interno entre los centros sin UCI o Unidad Coronaria y los que si disponen de dichas unidades, tiempo que llega a triplicarse. La independencia del 
médico de urgencias en tomar la decisión y administrar el tratamiento, también queda patente en una disminución drástica de los tiempos de administración del tratamiento trombolítico. Este hecho ha quedado tambien reflejado por otros autores. En el estudio GUSTO Time to Treatment substudy (12) se apreció que la consulta al cardiologo incrementaba en 15 minutos el tiempo medio para el tratamiento. Así mismo cuando el paciente es trasladado de un área del hospital a otra para realizar el tratamiento trombolítico,

\section{Bibliografía}

1. The GUSTO Angiographic Investigators. The effects of tissue plasminogen activator, streptokinase, or both on coronary-artery patency, ventricular function and survival after acute myocardial infarction. $\mathrm{N}$ Engl J Med 1993; 329: 1615-22.

2. Kudenchuk PJ, Litwin PE, Dewhurst TA, Ho MT, Martin JS, Weaver WD for the MITI investigators. Early predictors of hospital mortality in the acute myocardial infarction (resumen). J Am Coll Cardiol 1992;19 Supl. A:153.

3. National Heart Attack Alert Program Coordinating Committee, 60 minutes to treatment working group. Emergency department: rapid identification and treatment of patients with acute myocardial infarction. Ann Emerg Med 1994; 23: 311-29.

4. Loma Osorio-A, Garcia-Castrillo L, Arós F, Lopetegui P, Recuerda E, Epelde F. El infarto agudo de miocardio en los servicios de urgencias españoles. El estudio EVICURE. Rev Esp Cardiol 2002; 55: 1098-100.

5. Ochoa Gomez FJ, Carpintero Escudero JM, Ramalle-Gomara E, Lisa Catón V, Marco Aguilar P. Causas de exclusión de tratamiento trombolítico en el infarto agudo de miocardio. An Med Interna (Madrid) 1998; $80-2$.

6. ACC/AHA Guidelines for the management of patients with acute myocardial infarction. Executive Summary. Circulation 1996; 94: 2341-50. también se asiste a un incremento significativo del tiempo de demora. Revisando los protocolos de actuación de nuestros centros y dejando independencia de administración del tratamiento trombolítico en el IAM podríamos mejorar de manera ostensible el tiempo puerta-aguja.

Limitaciones del estudio: se trata de es una encuesta en la cual los hospitales ofrecieron sus datos de manera retrospectiva. Sería necesario a raiz de los datos encontrados realizar un estudio prospectivo para confirmarlos.

7. Heras M, Fernández-Ortíz A, Gómez Guindal J, Iriarte JA, Lidón RM, Pérez Gómez F, et al. Guías de actuación clínica de la Sociedad Española de Cardiología. Recomendaciones para el uso del tratamiento antitrombótico en cardiología. Rev Esp Cardiol 1999; 52: 801-20.

8. Cabadés A, López Bescós L, Arós F, Loma-Osorio A, Bosch X, Pabón $\mathrm{P}$, et al. Variabilidad en el manejo y pronóstico a corto y medio plazo del infarto de miocardio en España: el estudio PRIAMHO. Rev Esp Cardiol 1999; 52: 767-75.

9. Kereiakes DJ, Weaver WD, Anderson JL, Feldman T, Gibbler B, Aufderheide T, et al. Time delays in the diagnosis and treatment of acute myocardial infarction:a tale of eight cities. Am Heart J 1990; 120: 773-80.

10. Birkhead JS. Time delays in the provision of thrombolytic treatment in six distirct hospitals. BMJ 1992; 305: 445-8.

11. Rawles $\mathbf{J}$ on behalf of the GREAT group. Halving of mortality at 1 year by domiciliary thrombolysis in the Grampian region early anistreplase trial (GREAT). J Am Coll Cardiol 1994; 23: 1-5.

12. Kline EM, Smith DD, Martin JS, Dracup K, Woodfield LH, Granger $\mathrm{CB}$, et al. In Hospital treatment delays in patients treated with thrombolytic therapy: a report of the GUSTO Time Treatment Substudy (resumen). Circulation 1992; 86 (Supl. 1): 702. 